\title{
Modulating pain thresholds through classical conditioning
}

\author{
Juliane Traxler $^{\text {Corresp., }}{ }^{1,2}$ ， Victoria J. Madden $^{3}{ }^{\text {, G. Lorimer Moseley }}{ }^{4}$, Johan W.S. Vlaeyen ${ }^{1,2}$ \\ 1 Research Centre for Health Psychology, KU Leuven, Leuven, Belgium \\ 2 \\ 3 Department of Psychiatry and Mental Health, Department of Anaesthesia and Perioperative Medicine, University of Cape Town, Cape Town, South Africa \\ 4 Body in Mind Research Group, University of South Australia, Adelaide, Australia \\ Corresponding Author: Juliane Traxler \\ Email address: juliane.traxler@kuleuven.be
}

Background. Classical conditioning has frequently been shown to be capable of evoking fear of pain and avoidance behaviour in the context of chronic pain. However, whether pain itself can be conditioned has rarely been investigated and remains a matter of debate. Therefore, the present study investigated whether pain threshold ratings can be modified by the presence of conditioned non-nociceptive sensory stimuli in healthy participant.

Methods. In 51 healthy volunteers, pain threshold to electrocutaneous stimuli was determined prior to participation in a simultaneous conditioning paradigm. Participants underwent an acquisition phase in which one non-painful vibrotactile stimulus $\left(\mathrm{CS}^{+}\right)$was repeatedly paired with a painful electrocutaneous stimulus, whereas a second vibrotactile stimulus of the same quality and intensity (CS) was paired with a non-painful electrocutaneous stimulus. Stimulation was provided on the lower back with close proximity between the CSs and the US. In the test phase, electrocutaneous stimuli at the individually-set threshold intensity were simultaneously delivered together with either a $\mathrm{CS}^{+}$or $\mathrm{CS}^{-}$. Pain intensity ratings were obtained after each trial; expectancy ratings were obtained after each block. The primary outcome was the percentage of test stimuli that were rated as painful.

Results. Test stimuli were more likely to be rated as painful when they were paired with the $\mathrm{CS}^{+}$than when they were paired with the CS. This effect was not influenced by contingency awareness, nor by expectancies or mood states.

Discussion. The findings support the notion that the judgement of an event being painful or non-painful can be influenced by classical conditioning and corroborate the possible role of associative learning in the development and maintenance of chronic pain. 


\section{Modulating Pain Thresholds through Classical Conditioning}

2

3 Juliane Traxler ${ }^{\text {ab* }}$, Victoria J. Madden ${ }^{\mathrm{c}}$, G. Lorimer Moseleyd, Johan W.S. Vlaeyen ${ }^{\mathrm{ab}}$

4

5

6 a Research Centre for Health Psychology, University of Leuven, Leuven, Belgium

7 b Experimental Health Psychology, Maastricht University, Maastricht, The Netherlands

$8 \quad$ c Department of Psychiatry and Mental Health and Department of Anaesthesia and Perioperative

9 Medicine, University of Cape Town, South Africa.

$10{ }^{\mathrm{d}}$ Body in Mind Research Group, University of South Australia, Adelaide, Australia.

11

12

* Corresponding author. Address: Department of Psychology, University of Leuven, Tiensestraat

102, Box 3726, 3000 Leuven, Belgium. Phone: +32 163281 68; E-mail:

15 juliane.traxler@kuleuven.be. 


\section{Abstract}

18

Classical conditioning has frequently been shown to be capable of evoking fear of pain and avoidance behaviour in the context of chronic pain. However, whether pain itself can be conditioned has rarely been investigated and remains a matter of debate. Therefore, the present study investigated whether pain threshold ratings can be modified by the presence of conditioned non-nociceptive sensory stimuli in healthy participants. In 51 healthy volunteers, pain threshold to electrocutaneous stimuli was determined prior to participation in a simultaneous conditioning paradigm. Participants underwent an acquisition phase in which one non-painful vibrotactile stimulus $\left(\mathrm{CS}^{+}\right)$was repeatedly paired with a painful electrocutaneous stimulus, whereas a second vibrotactile stimulus of the same quality and intensity $\left(\mathrm{CS}^{-}\right)$was paired with a non-painful electrocutaneous stimulus. Stimulation was provided on the lower back with close proximity between the CSs and the US. In the test phase, electrocutaneous stimuli at the individually-set threshold intensity were simultaneously delivered together with either a $\mathrm{CS}^{+}$or $\mathrm{CS}^{-}$. Pain intensity ratings were obtained after each trial; expectancy ratings were obtained after each block. The primary outcome was the percentage of test stimuli that were rated as painful. Test stimuli were more likely to be rated as painful when they were paired with the $\mathrm{CS}^{+}$than when they were paired with the CS- This effect was not influenced by contingency awareness, nor by expectancies or mood states. The findings support the notion that the judgement of an event being painful or non-painful can be influenced by classical conditioning and corroborate the possible role of associative learning in the development and maintenance of chronic pain. 


\section{Introduction}

The development of chronic pain, i.e. the persistence of pain long after tissue healing, is

41

42

not well understood. A proposed mechanism in the transition from acute to chronic pain that seems intuitive and is widely accepted among clinicians (Madden \& Moseley 2016) is associative learning in the form of classical conditioning. It is assumed that during acute pain, nociceptive signalling serves as the unconditioned stimulus (US), and occurs alongside non-nociceptive signalling such as touch, proprioception and haptic perception, which serves as the conditioned stimulus (CS), such that the association of both stimuli is stored in memory. According to this model, the CS then carries information about the occurrence of the US: a stimulus repeatedly paired with pain $\left(\mathrm{CS}^{+}\right)$indicates danger and elicits the expectancy of pain, while a stimulus paired with non-painful sensations $\left(\mathrm{CS}^{-}\right)$indicates safety (Domjan 2005). By virtue of the CS-US association, CS's may then instil conditioned responses (CR) such as increases in muscle tension, sympathetic activation, avoidance behaviour or fear in the absence of the US (Linton et al. 1984). Some theorists have espoused the idea that pain itself can occur as a CR which continues to maintain itself after the initial source of nociception has subsided (Flor \& Birbaumer 1994; Linton et al. 1984; Moseley \& Vlaeyen 2015). Accordingly, classical conditioning might evoke and maintain chronic pain.

While it has repeatedly been shown that various pain-related constructs including expectancies, pain-related fear, fear of movement and avoidance behaviour can be classically conditioned (Claes et al. 2016; Gramsch et al. 2014; Jepma \& Wager 2015; Meulders et al. 2011; Peuterl et al. 2011; Zaman et al. 2015), less is known about whether pain itself can be conditioned as well. Only a few attempts have been made to elicit pain as a classically conditioned response (for a review, see Madden et al. 2015). So far, most studies evaluated conditioned hyperalgesia 
62 (increased painful response), and provided strong support that pain can indeed be amplified 63 through associative learning, as identical nociceptive stimuli were found to evoke different 64 perceptions merely depending on established, frequently unconscious associations (Harvie et al. 65 2016a; Jensen et al. 2015). In contrast, only three studies indirectly investigated conditioned allodynia (a painful response upon non-nociceptive stimuli) and reported contradictory findings (Bräscher et al. 2017; Klinger et al. 2010; Williams \& Rhudy 2007). Examining this topic further, Madden and colleagues conducted two targeted experiments using laser-stimuli (Madden et al. 2016a) or thermal stimuli (Madden et al. 2016b), both of which activate nociceptive A $\delta$ - and Cfibres, as US and vibrotactile stimuli activating non-nociceptive A $\beta$-fibres (Abraira \& Ginty 2013) as CSs to specifically test the effect of classical conditioning on pain threshold ratings. At-painthreshold stimuli are considered to be arbitrary, meaning they are perceived as painful or nonpainful at chance level. It was hypothesized that the classical conditioning procedure would lead to a higher percentage of these at-pain-threshold stimuli being perceived as painful, signifying a decrease in pain threshold. However, only the former study (Madden et al. 2016a) demonstrated a decrease in pain thresholds on $\mathrm{CS}^{+}$trials whereas the latter (Madden et al. 2016b) did not. It was

77 speculated that these inconsistent findings could be due to the different types of USs used, as laser stimuli produce more discrete sensations than contact heat stimuli, allow for faster temperature increases, and selectively activate $\mathrm{A} \delta$ - and C-fibre nociceptors whereas thermodes used for thermal stimulation concomitantly activate low threshold mechanoreceptors (Plaghki \& Mouraux 2003).

81 Consequently, studies on the conditioning of allodynia and pain thresholds are scarce and their findings are mixed at best. In order to better understand and extend previous findings, further

83 research is required. 
84

85

86

87

88

89

90

91

92

93

94

95

96

97

98

The present study aimed at examining whether the judgment of stimuli as painful or nonpainful can be a classically conditioned response in healthy participants by replicating and extending the studies by Madden et al. (Madden et al. 2016a; Madden et al. 2016b). Similar to these studies, in the present experiment two neutral vibrotactile stimuli $\left(\mathrm{CS}^{+}, \mathrm{CS}^{-}\right)$were paired with a painful $\left(\mathrm{US}_{\mathrm{P}}\right)$ and a non-painful $\left(\mathrm{US}_{\mathrm{NP}}\right)$ electrocutaneous stimulus, respectively, all of which were applied to the lower back. The USs evoked an unconditioned response (URs), namely reported pain. Subsequently, both $\mathrm{CS}^{+}$and $\mathrm{CS}^{-}$were presented simultaneously with at-painthreshold electrocutaneous stimuli $\left(\mathrm{US}_{\mathrm{PT}}\right)$. It was expected that these $\mathrm{US}_{\mathrm{PT}}$, $\mathrm{s}$ would be experienced and rated as painful as opposed to non-painful $(\mathrm{CR})$ on more of the $\mathrm{CS}^{+}$trials than the $\mathrm{CS}^{-}$trials, confirming that the judgment of stimuli as painful or non-painful can be modulated through classical conditioning. The main difference with the previous studies was the type of US used, namely an electrocutaneous stimulus instead of laser stimulation (Madden et al. 2016a) or thermal stimulation (Madden et al. 2016b). This type of stimulation was chosen as it shares some of the features of laser stimuli, namely the discreteness and speed with which activation of nociceptors is achieved, while co-activating mechanoreceptors like thermal stimuli. Thus, although not directly comparing the different types of USs, the present study may help to interpret previous contradictory findings.

\section{Participants}

As the present experiment closely resembled the study by Madden, Bellan, et al. (2016a), an a priori sample size calculation using $\mathrm{G}^{*}$ Power [8] was conducted with the effect size obtained by Madden, Bellan, et al. $(2016 a)(E S=0.58)$, according to which 34 participants would need to 
107 be included for a power of $.95(\alpha=0.05)$. In order to deal with a potentially overestimated effect

108 size of the original study (Brandt et al. 2014), the study aimed at including 50 participants in total.

109 Participants were recruited at the University of Leuven, Belgium, using flyers, posters, word of

110 mouth, and through an online platform for participant recruitment.

111 Participants were eligible for participation if they were older than 18 years, proficient in

112 the Dutch language and able to consent autonomously. Exclusion criteria based on self-report were

113 (a) a history of chronic pain (defined as pain on most days for 3 months or longer), (b) acute pain

114 at the time of testing, (c) use of analgesic medication on the day of testing, (d) use of medication

115 that could alter skin sensitivity or healing, (e) a skin condition that would not allow to tolerate

116 electrode application without damage (e.g., a past operation at the location where the electrodes

117 would be placed), (f) sensation problems, (g) a serious medical condition (e.g., diabetes mellitus,

118 peripheral vascular disease, cardiovascular disease, neurological problems), (h) pregnancy, (i) use

119 of an electronic implant (e.g., a pacemaker) or (j) a previous or current psychiatric diagnosis.

120 Furthermore, participants were excluded mid-procedure if they (1) were unable to discriminate the

121 locations of the vibrotactile stimuli during baseline $(<50 \%$ correct $)$, and/or $(2)$ reported a pain

122 threshold that was too high or too low for the equipment's technical and safety settings.

Written informed consent was obtained from each participant prior to testing, and

participation was rewarded with course credit or with the chance of winning a weekend trip worth

G-2016 11 663).

\section{Stimuli}



means of two reusable Coulbourn electrodes $(4 \mathrm{~mm})$ that were attached $5 \mathrm{~mm}$ apart from each other on participants' lower back and were controlled by a constant-current stimulator (DS7, Digitimer Limited, Hertfordshire, UK). Single pulses as well as 3-pulse, 5-pulse and 7-pulse trains were used, with a duration of $0.5 \mathrm{~ms}$ each, and an inter-stimulus interval of $5 \mathrm{~ms}$. Using pulse trains allows for the production of more stable stimulus intensities (Mouraux et al. 2013; Mouraux et al. 2014). Due to this effect, a lower electric current was necessary to evoke a painful sensation, which improved the signal-to-noise ratio and reduced the risk of causing burns to the skin (Inui et al. 2002). Stimulus intensities were determined by first determining individual pain thresholds using a 5-pulse train. This was called the pain-threshold intensity. The non-painful stimuli (US $\left.\mathrm{NP}_{\mathrm{NP}}\right)$ were single pulse stimuli at the pain-threshold intensity and the painful stimuli $\left(\mathrm{US}_{\mathrm{P}}\right)$ were 7-pulse trains at the pain-threshold intensity. During the test phase, 3-pulse trains served as at-painthreshold stimuli $\left(\mathrm{US}_{\mathrm{PT}}\right)$ instead of 5-pulse trains in order to account for sensitization and for perceived control during the calibration phase as participants calibrated the pain threshold intensity themselves by means of clicking on "more" or "less" buttons displayed on the computer screen. The output of this was visible for the experimenter who adjusted the stimulus intensity on the DS7 accordingly. All choices about pulse-trains and calibration procedure were based on a pilot study that is described in the section Procedure. on the left side of the lower back. One tactor (cephalad/“upper”) was placed $40 \mathrm{~mm}$ above the electrodes, one (caudad/"lower") was placed $40 \mathrm{~mm}$ below the electrodes, and one was attached 
$15317 \mathrm{~ms}$. Either the caudad or cephalad tactor was conditioned to be $\mathrm{CS}^{+}$by repeatedly being paired 154 with a painful electrocutaneous stimulus $\left(\mathrm{US}_{\mathrm{P}}\right)$, while the other became the $\mathrm{CS}^{-}$by being paired 155 with a non-painful electrocutaneous stimulus (US $\left.\mathrm{UP}_{\mathrm{NP}}\right)$. The onset of the two stimuli 156 (electrocutaneous + vibrotactile) occurred simultaneously at all times. Allocation of tactor to $\mathrm{CS}^{+}$ 157 or $\mathrm{CS}^{-}$was counterbalanced across participants in a randomized order. The lateral tactor was only 158 used for calibration purposes and remained at that (neutral) location during the whole experiment.

\section{Measures and Outcomes} Manipulation Check. In order to check whether the conditioning procedure was successful, US expectancy ratings were conducted at several time points during the study (see Procedure). Participants were required to answer the questions (i) "To what extent do you expect the stimulus package to be painful (rather than non-painful) if the stimulus package includes the upper vibration?" and "To what extent do you expect the stimulus package to be painful (rather than non-painful) if the stimulus package includes the lower vibration?" on a numerical rating scale (NRS) ranging from $0=$ "I do not expect that it will feel painful" to $10=$ "I fully expect that it will feel painful".

\section{Positive and Negative Affect.} different emotional states (e.g. guilty, inspired, restless) are currently applicable to them on a 5point Likert scale $(1=$ not at all, $5=$ very much). The two subscales have good psychometric

175 properties, including good convergent and discriminant validity (Crawford \& Henry 2004). In the 
176 present sample, there was a high internal consistency for the positive affect subscale $(\alpha=.88)$.

177 However, that for the negative affect subscale was rather low $(\alpha=.60)$.

178

$179 \quad$ Participant perceptions.

At the end of the experiment, participants answered a number of questions in order to assess

181

182

183

184

185

186

187

188

189

190

191

192

193

194

195

196

197 198

(1) whether they were naïve to the purpose of the experiment (i.e. blinding check), (2) whether they perceived differences between the vibrations of the two CS tactors, (3) how they perceived the timing of the vibrations relative to the electrocutaneous stimuli, (4) whether they noticed a relationship between the vibrotactile CSs and the painfulness of the electrocutaneous USs (i.e. contingency awareness/awareness of the conditioning paradigm), and (5) whether they experienced a direct association between their adaptation of the stimulus intensity and the actual intensity during the calibration phases.

\section{Outcome.}

The primary outcome of this study was the percentage of trials during test phase in which at-pain-threshold electrocutaneous stimuli (US $\mathrm{PT}_{\mathrm{PT}}$ at test phase) were experienced as painful, when the $\mathrm{CS}^{+}$was active, as compared to when the $\mathrm{CS}^{-}$was active. For that purpose, participants indicated whether or not each 'stimulus package', i.e. the combination of either vibration with the electrocutaneous stimulation, was painful or non-painful and rated the intensity of the stimuli on the Sensation and Pain Rating Scale (SPARS, initially called the FESTNRS; Madden et al. 2016b) within a maximum of 15 seconds after stimulus onset. The SPARS captures both painful and nonpainful experiences as it runs from $-50=$ "no sensation", through $0=$ pain threshold ("the exact point at which you feel transition to pain") to $+50=$ "most intense pain you can imagine". 
199 Participant ratings on the SPARS show a curvilinear relationship to stimulus intensity, with a

200 slightly steeper slope at the extremes of the scale and stable stimulus-response characteristics

201 across the range of the scale (Madden et al. April, 2018). A visual scale showing the range and the

202 anchors was presented on a computer screen on which participants could select any number within

203 this range. Furthermore, participants reported which tactor they felt (upper vs. lower) during each

204 trial by clicking one of two buttons.

205

206

207

The procedure of this study was adapted from Madden et al. (2016a). The study has a

208

209

210

211

212

213

214

215

216

217

218

219

220

221

\section{Procedure} within-subject design and consists of four main phases (see also Fig. 2). The protocol has been locked on the online database Augias from KU Leuven from which we did not deviate.

\section{Pilot study.}

A pilot study with 17 participants was conducted in order to test and optimize the methods and procedures to be used in the full-scale experiment. The general procedure did not have to be changed and can be found below. However, the ideal number of pulse trains for delivering nonpainful, at-pain-threshold and painful stimuli had to be tested resulting in a 1-4-7 grading, which provided easily differentiable intensities.

Importantly, pain thresholds proved very unstable, with both habituation and sensitization occurring throughout the experiment. In order to counteract this, recalibration phases were included between acquisition and test blocks. Regarding sensitization, participants were asked to (re-)calibrate to -5 instead of 0 so as to ensure that the stimulus would be closer to just non-painful rather than to just painful. Additionally, it was decided to use a 3-pulse train as the at-painthreshold stimulus $\left(\mathrm{US}_{\mathrm{PT}}\right)$ in the test phase because the 5-pulse train which had been calibrated to 
$222-5$ was consistently rated as painful during testing. We also tried to minimize context changes

223 between (re-)calibration phases and test phases. However, given the frequent (re-) calibrations, a

224 fast and precise calibration procedure was needed. The pilot study showed that self-adjustment

225 through the participant by means of clicking "more" and "less" buttons displayed on a computer

226 screen was more efficient than the commonly used adaptive staircase procedure (e.g. Madden et

227 al. 2016a).

228 A final aspect that was addressed in the pilot study was the intensity and distance between

229 the tactors. The distance between the tactors was increased from $70 \mathrm{~mm}$ (Madden et al. 2016a) to

$23080 \mathrm{~mm}$ and the vibration intensity was calibrated to a level that would permit good

231 distinguishability.

232 Preparation.

233 Prior to testing, participants received written information about the study without details

234 about the study goals and exact procedures so as to ensure blinding. Participants were screened for

235 exclusion criteria via e-mail. Upon arrival at the lab, participants once more received general study

236 information. They had the opportunity to ask questions and were instructed to carefully read and

237 sign the informed consent form and an exclusion criteria form afterwards. Participants were seated

238 in front of a computer, straddling a chair. Before the electrodes were attached on the left side of

239 the lower back, participants crossed their arms over their chest, looked upwards, and bent

240 backwards. The line of greatest curvature or 'hinge' was identified. Moving along this line, the

241 two electrodes were taped to the skin $40 \mathrm{~mm}$ left of the spine once the participant had resumed a

242 comfortable resting posture. The tactors were placed $40 \mathrm{~mm}$ above, $40 \mathrm{~mm}$ below and $15 \mathrm{~mm}$ to

243 the left of the electrodes.

$244 \quad$ Calibration phase. 

$\mathrm{mA}$ stimulus paired with the lateral tactor as a neutral vibrotactile stimulus and were asked to rate their experience. Next, they were instructed to adjust the stimulus intensity to match their personal ratings of $-30,-10,+10,+30$ and finally 0 (threshold) by clicking on "more" or "less" buttons displayed on the computer screen. All electrocutaneous stimuli in the calibration phase were delivered simultaneously with the lateral tactor. Next, participants received and rated six electrodetactor combinations with intensities between those previously determined as -30 and +30 , and for anticipated perceptual sensitization) and was subsequently used for all single pulses and pulse trains.

\section{Baseline phase.} received ten vibration-only trials, i.e. the upper and lower tactor were activated five times each, rated each trial on the SPARS and indicated which tactor was activated. Participants who were unable to discriminate the tactor locations $(<50 \%$ correct) were excluded at this point in the procedure. Subsequently, participants provided expectancy ratings.

\section{Acquisition phase.}

During the acquisition phase, participants received twelve painful electrocutaneous stimuli

(7-pulse trains $-\mathrm{US}_{\mathrm{P}}$ ) paired with the tactor that was assigned to become the $\mathrm{CS}^{+}$. Similarly, the other tactor $\left(\mathrm{CS}^{-}\right)$was paired with the non-painful electrocutaneous stimulus (single pulses - 
268 ordered with no more than two identical trials delivered successively. The reinforcement 269 contingency was set to $100 \%$. Participants were instructed to rate each stimulus package on the

270 SPARS. After each block, participants were asked to give expectancy ratings and to readjust the

271 intensity of the 5-pulse trains (in combination with the lateral tactor) to -5 in order to counteract

272 habituation and sensitization. These newly determined at-pain-threshold intensities were then used

273 as stimulus intensity in the subsequent block.

274

275

276

277

278

279

280

281

282

283

284

285

286

287

\section{Test phase.}

In total, the test phase consisted of three blocks of 36 trials each (108 trials in total) with the following stimulus packages being delivered: $15 \mathrm{CS}^{+} / \mathrm{US}_{\mathrm{PT}}$ trials and $15 \mathrm{CS}^{-} / \mathrm{US}_{\mathrm{PT}}$ trials as main outcomes, $30 \mathrm{CS}^{+} / \mathrm{US}_{\mathrm{P}}$ trials and $30 \mathrm{CS}^{-} / \mathrm{US}_{\mathrm{NP}}$ trials as reinforcement in order to prevent extinction, and $9 \mathrm{CS}^{+}$-only trials and $9 \mathrm{CS}^{-}$-only trials. These stimulus packages were delivered in random order with no more than two identical trials being delivered successively. After each trial participants provided intensity ratings on the SPARS and indicated the tactor location. Again, they gave expectancy ratings and readjusted the intensity of the 5-pulse trains to -5 between blocks.

Furthermore, after the final test block, participants were instructed to adjust the intensity of the 5pulse train to their pain threshold ( 0 on SPARS) two times, one time in combination with the upper tactor and the other time with the lower tactor. Whether recalibration was done with the upper or the lower tactor first was counterbalanced across participants.

Finally, participants filled in the PANAS and the five questions about their perceptions of the study. After this, the electrodes and vibrotactors were removed. Participants were debriefed, assigned their course credit and thanked for their participation.

\section{Statistical Analysis}


following packages were used: readr (Wickham et al. 2017), magrittr (Bache \& Wickham 2014),

294

295

296 tidyverse (Wickham 2017), and ggplot2 (Wickham 2009).

Prior to analysis, data were checked for normality, sphericity, outliers and missing values. The only missing values were found for the indication of tactor localisation. These were omitted for the analyses on tactor localisation mistakes. When appropriate, non-parametric tests were applied. Follow-up pairwise comparisons with Bonferroni adjustment were applied to ANOVAs with significant results.

To rule out any baseline differences in vibration intensities of the two tactors, a Wilcoxon signed rank test was conducted on baseline intensity ratings, with Condition $\left(\mathrm{CS}^{+}\right.$vs. $\left.\mathrm{CS}^{-}\right)$as within-subject factor. To test whether manipulations were successful, expectancy ratings as well as intensity ratings were analysed by means of two separate 2 (Condition: $\left.\mathrm{CS}^{+} \mathrm{vs}^{\mathrm{C}} \mathrm{CS}^{-}\right)$x 3 (Phase: baseline, acquisition, test) repeated measures (RM) ANOVAs. Additionally, blinding for the study goal, contingency awareness for the CS-US pairings, perceived control over the stimulus calibrations as well as quality of the vibrotactile stimuli and simultaneity of the vibrotactile and electrocutaneous stimuli were checked.

Subsequently, in order to test the main hypothesis, a binary variable reflecting non-painful $(\leq 0)$ and painful $(>0)$ at-pain-threshold trials was computed, based on which a second variable reflecting the percentage of $U_{S_{P T}}$ trials rated as painful was created. Next, the percentage of US $\mathrm{PT}_{\mathrm{PT}}$ trials rated as painful was compared across the two conditions, i.e. between the $15 \mathrm{CS}^{+} / \mathrm{US}_{\mathrm{PT}}$ and $15 \mathrm{CS}^{-} / \mathrm{US}_{\mathrm{PT}}$ stimulus packages, by means of a Wilcoxon signed rank test - this was the primary analysis. As secondary analyses, a 2 (Condition: $\mathrm{CS}^{+} / \mathrm{US}_{\mathrm{PT}}$ Vs. $\mathrm{CS}^{-} / \mathrm{US}_{\mathrm{PT}}$ ) x 3 (Test Block: 1, 2, 3) 
314 RM ANOVA was conducted to examine time effects, and a Wilcoxon signed rank test was 315 conducted to compare the intensity ratings for the $\mathrm{CS}^{+} / \mathrm{US}_{\mathrm{PT}}$ and $\mathrm{CS}-/ \mathrm{US}_{\mathrm{PT}}$ trials. As additional 316 measures of shifts in perception, two Wilcoxon signed rank tests were used to (1) compare the 317 intensity ratings for the $\mathrm{CS}^{+}$-only and $\mathrm{CS}^{-}$-only trials and (2) compare the recalibrated at-pain318 threshold intensities (from the final re-calibration phase) when paired with the $\mathrm{CS}^{+}$compared to

$319 \mathrm{CS}^{-}$. For the latter analysis, the recalibrated intensities for the $\mathrm{CS}^{+}$and $\mathrm{CS}^{-}$were divided by the 320 respective group mean recalibrated intensities. In order to control for mistakes on the tactor 321 localisation task, these analyses were repeated with perceived rather than actual tactor location, 322 i.e. trials on which the $\mathrm{CS}^{+}$location was wrongly perceived as the $\mathrm{CS}^{-}$location were considered $323 \mathrm{CS}^{-}$trials in the analyses, and vice versa. Lastly, the relationship between the primary outcome and 324 negative affect (PANAS), contingency awareness, and expectancy, were explored by means of 325 analyses of covariance (ANCOVAs), with total scores of the positive and negative subscales of 326 the PANAS, the binary variable contingency awareness (yes/no) and the expectancy ratings given 327 for the upper and lower tactor during test phase.

Results

Fifty-one participants ( 7 males) aged 18 to 31 years $(\mathrm{M}=19.39, \mathrm{SD}=2.55)$ were included.

331 Two participants were excluded mid-procedure for having no clear non-painful range and 332 perceiving almost all of the stimuli as painful. Four other participants were excluded due to 333 technical problems that may have rendered their data unreliable. None were excluded dor inability 334 to discriminate between the vibrotactile stimuli. Consequently, the analyses were conducted on the 335 final sample of 45 participants (6 males). Participant characteristics are described in Table 1. The mean intensity of at-pain-threshold stimuli at the initial calibration was $3.18 \mathrm{~mA}(\mathrm{SD}=1.945)$. 
Table 1.

339

\section{Blinding and Contingency Awareness}

At the completion of the experiment, 40/45 participants were unaware of the goal of the

study and 17 were unaware of the contingency, i.e. either they reported to have noticed no

correlation at all between the location of the vibration and whether the electrocutaneous stimulus

was painful or not, or they identified the $\mathrm{CS}^{-}$to be associated with the painful stimuli. Fourteen

participants experienced differences between the vibrations from the upper and the lower tactor,

ten of whom perceived the $\mathrm{CS}^{+}$as "stronger", "sharper", and "longer" and four of whom did not

specify the perceived difference. Four participants indicated that the US and CS did not have a

simultaneous onset but that they perceived either the vibrotactors $(n=3)$ or the electrocutaneous stimulus $(n=1)$ first. Lastly, 29 participants reported that they experienced control over the calibration whereas 16 reported that they did not.

\section{Manipulation Checks}

There was initially no significant difference between the intensity ratings for the two vibrotactors at baseline (Wilcoxon signed-rank test: $Z=-1.084 ; p=.278 ; \mathrm{CS}^{+}=-32.22 ; \mathrm{CS}^{-}=$two vibrotactors (Wilcoxon signed-rank test: $Z=-1.302 ; p=.193 ; \mathrm{CS}^{+}=1.93 ; \mathrm{CS}^{-}=-1.51$ ). Expectancy ratings for $\mathrm{CS}^{+}$and $\mathrm{CS}^{-}$across the three phases are depicted in Fig. 3 . trials (main effect of Condition, $F(1,44)=225.148 ; p<.001 ; \eta_{\mathrm{p}}^{2}=.837 ; 95 \% \mathrm{CI}=[23.062$; 
360

361

362

363

364

365

366

367

368

369

370

371

372

373

374

375

376

377

378

379

380

381

382

30.218]; $\left.\mathrm{CS}^{+} / \mathrm{US}_{\mathrm{P}}: M=9.630, S E=1.024 ; \mathrm{CS}^{-} / \mathrm{US}_{\mathrm{NP}}: M=-17.010, S E=1.434\right)$ and this increase in intensity ratings was greater for $\mathrm{CS}^{+} / \mathrm{US}_{\mathrm{P}}$ trials than for $\mathrm{CS}^{-} / \mathrm{US}_{\mathrm{NP}}$ trials (Phase $\mathrm{x}$ Condition interaction, $\left.F(1,44)=11.901, p=.001 ; \eta_{\mathrm{p}}{ }^{2}=.213\right)$. Follow-up pairwise comparisons indicated that intensity ratings for $\mathrm{CS}^{+} / \mathrm{US}_{\mathrm{P}}$ trials and $\mathrm{CS}^{-} / \mathrm{US}_{\mathrm{NP}}$ trials increased from acquisition phase to test phase $(p<.001)$.

In order to check whether the classical conditioning procedure was successful, a 2 (Condition: $\mathrm{CS}^{+}$vs $\mathrm{CS}^{-}$) x 3 (Phase: baseline, acquisition, test) RM ANOVA was performed on expectancy ratings. This analysis revealed a significant Condition $\mathrm{x}$ Phase interaction $(F(2,43)=$ 14.927, $p<.001 ; \eta_{\mathrm{p}}{ }^{2}=.410$ ): follow-up pairwise comparisons revealed that expectancy ratings for both $\mathrm{CS}^{+}$and $\mathrm{CS}^{-}$increased significantly from post-baseline to post-acquisition as well as from post-acquisition to post-test (both $p<.001$ ). Finally, participants who were contingency aware showed a greater increase over time in the difference between expectancy ratings for $\mathrm{CS}^{+}$and $\mathrm{CS}^{-}$ (see Fig. 3; Condition x Phase x Contingency Awareness interaction, $F(2,42)=9.372, p<.001$; $\left.\eta_{\mathrm{p}}^{2}=.308\right)$

\section{Main Hypothesis: Effects on Intensity Ratings of Pain Threshold Stimuli}

Pain intensity ratings for $\mathrm{CS}^{+} / \mathrm{US}_{\mathrm{P}}$ and $\mathrm{CS}^{-} / \mathrm{US}_{\mathrm{NP}}$ trials, for $\mathrm{CS}^{+} / \mathrm{US}_{\mathrm{PT}}$ and $\mathrm{CS}^{-} / \mathrm{US}_{\mathrm{PT}}$ trials as well as for $\mathrm{CS}^{+}$only and $\mathrm{CS}^{-}$only trials are presented in Table 2 . The test of the current study's main hypothesis showed that participants rated more $\mathrm{US}_{\mathrm{PT}}$ trials as painful when they were paired with the $\mathrm{CS}^{+}(81.5 \%, S D=21.03)$ than when they were paired with the $\mathrm{CS}^{-}(72.7 \%, S D=24.69$; Wilcoxon signed rank test: $Z=-3.033 p=.002)$. In absolute numbers, $12.22(S D=3.154)$ out of $15 \mathrm{CS}^{+} / \mathrm{US}_{\mathrm{PT}}$ trials were rated as painful compared to $10.91(S D=3.704)$ of the $15 \mathrm{CS}^{-} / \mathrm{US}_{\mathrm{PT}}$ trials. This difference was still apparent when tactor localisation mistakes were controlled for (83.05\% 
$383[S D=19.22]$ of $\mathrm{CS}^{+} / \mathrm{US}_{\mathrm{PT}}$ trials vs $70.24 \%[S D=26.29]$ of $\mathrm{CS}^{-} / \mathrm{US}_{\mathrm{PT}}$ trials rated as painful, $Z=$ -

$3843.768 ; p<.001)$. The examination of time effects showed that participants rated more US $\mathrm{ST}_{\mathrm{PT}}$ trials 385 as painful when they were paired with the CS+ than when they were paired with the CS- across all 386 three blocks of the test phase (main effect of Condition, $F(1,44)=10.201 ; p=.003 ; \eta_{\mathrm{p}}{ }^{2}=.188$ ), 387 and that this difference decreased over the three blocks of the test phase (main effect of Block $388\left(F(2,43)=3.360 ; p=.044 ; \eta_{\mathrm{p}}^{2}=.135 ;\right.$ no Block x Condition interaction, $\left.F(2,43)=.332 ; p=.719\right)$. 389

Table 2.

391

\section{Secondary Analyses: Effects on Intensity Ratings}

Participants rated $\mathrm{US}_{\mathrm{PT}}$ trials as significantly more painful when the $\mathrm{US}_{\mathrm{PT}}$ was paired with the $\mathrm{CS}^{+}(M=6.585, S D=5.128)$ than when the $\mathrm{US}_{\mathrm{PT}}$ was paired with the $\mathrm{CS}^{-}(M=4.113, S D=$ 4.989; Wilcoxon signed rank test: $Z=-3.302 ; p=.001$ ). This difference was still apparent when tactor localisation mistakes were controlled for $\left(Z=-3.515 ; p<.001 ; M_{C S+/ U S T}=6.881, S D=\right.$ 4.556; $\left.M_{C S-/ U S T}=3.779, S D=5.703\right)$.

Exact mean intensity ratings for $\mathrm{CS}^{+} / \mathrm{US}_{\mathrm{P}}$ and $\mathrm{CS}^{-} / \mathrm{US}_{\mathrm{NP}}$ trials during acquisition and test than in the acquisition phase. When examining the exact mean intensity ratings, a 2 (Condition: $\mathrm{CS}^{+} / \mathrm{US}_{\mathrm{P}} \mathrm{vs} \mathrm{CS}^{-} / \mathrm{US}_{\mathrm{NP}}$ ) x 2 (Phase: acquisition, test) repeated measures ANOVA showed that there was a significant Condition $\mathrm{x}$ Phase interaction effect $\left(F(1,44)=11.901 ; p=.001 ; \eta_{\mathrm{p}}^{2}=.213\right)$.

Pairwise comparisons revealed that intensity ratings differed significantly between acquisition and test phase $\left(M_{\text {acquisition }}=-6.120, S E=1.159 ; M_{\text {test }}=-1.259, S E=.874 ; \mathrm{p}<.001\right)$ as well as between 
405 the vibrotactors $\left(M_{C S+}=9.630, S E=1.024 ; M_{C S-}=-17.01, S E=1.434 ; \mathrm{p}<.001\right)$. None of these

406 results changed notably when contingency awareness was added to the analysis as a covariate.

407

408 Exploratory Analyses

409

Intensity ratings for the $\mathrm{CS}^{+}$-only and $\mathrm{CS}^{-}$-only trials did not differ between the two 410 conditions (Wilcoxon signed rank test: $Z=-1.155 ; p=.248$ ). This did not change when accounting

411 for tactor localisation mistakes on the CS-only trials $(Z=-1.307 ; p=.191)$. Recalibrated stimulus 412 intensities, i.e. for the 5-pulse train electrocutaneous stimulus paired with the lateral vibrotactor, 413 increased significantly from first (calibration phase) to last (before final test block) calibration 414 moment $(Z=-5.842 ; p<.001)$, with a mean intensity of $3.18 \mathrm{~mA}(S D=1.945)$ at first calibration 415 and $6.39 \mathrm{~mA}(S D=4.716)$ at the last recalibration. However, final recalibrations for $\mathrm{CS}^{+}$and $\mathrm{CS}^{-}$ $416 \operatorname{did}$ not differ $(Z=-.035 ; p=.972)$, with a mean intensity of $7.82 \mathrm{~mA}(S D=5.045)$ for the $\mathrm{CS}^{+}$and $4177.69 \mathrm{~mA}(S D=5.014)$ for the $\mathrm{CS}^{-}$.

The ANCOVAs revealed no effects of negative affect, contingency awareness, or expectancy ratings on the percentage of $\mathrm{CS}^{+}$and $\mathrm{CS}^{-}$trials rated as painful, on the intensity ratings of the $\mathrm{CS}^{+}$-only and $\mathrm{CS}^{-}$-only trials, or on the final recalibrations with $\mathrm{CS}^{+}$and $\mathrm{CS}^{-}$(all p-values $>$ $.05)$.

\section{Discussion}

The aim of the present study was to investigate whether simultaneous classical conditioning can modulate pain reports of at-pain-threshold stimuli. This knowledge may inform whether classical conditioning could be one of the mechanisms underlying the development or maintenance

427 of chronic pain. It was expected that, after an acquisition phase in which non-painful vibrotactile 
428 stimuli (CSs) were repeatedly paired with either painful or non-painful electrocutaneous stimuli,

$429 \mathrm{US}_{\mathrm{PT}}$, $\mathrm{S}$ would be experienced as painful on more of the $\mathrm{CS}^{+}$trials than the $\mathrm{CS}^{-}$trials, confirming

430 that pain threshold ratings can be modulated through classical conditioning.

431 The results confirmed our hypothesis: at-pain-threshold electrocutaneous stimuli evoked a

432 conditioned response, i.e. they were $6 \%$ more likely to be reported as painful and were rated on

433 average as more painful, if they were presented with $\mathrm{CS}^{+}$vibrotactile stimuli than if they were

434 presented with the $\mathrm{CS}^{-}$vibrotactile stimuli. Besides, the exact pain ratings for $\mathrm{CS}^{+} / \mathrm{US}_{\mathrm{PT}}$ trials were

435 significantly higher than those for $\mathrm{CS}^{-} / \mathrm{US}_{\mathrm{PT}}$ packages. Notably, the majority of CS-/US $\mathrm{PT}_{\mathrm{PT}}$ trials

436 were also perceived as painful. The calibration and classical conditioning procedures were

437 successful, as indicated by significant increases in intensity ratings across all three phases as well

438 as in pain expectancies from post-baseline to post-acquisition for the $\mathrm{CS}^{+}$but not the $\mathrm{CS}^{-}$, even

439 though many participants were not explicitly contingency aware. Neither the CS-only trials nor

440 the final recalibrations were significantly affected by classical conditioning. Recalibrated pain

441 threshold intensities increased throughout the experiment, indicating habituation.

The present findings are largely in line with the study by Madden et al. (2016a) and the

study by Williams \& Rhudy (2007), in that exposure to non-nociceptive vibrotactile stimuli was

444 found to influence pain thresholds through classical conditioning. The results specifically parallel

445 those by Madden et al. (2016a), as even the difference between intensity ratings for $\mathrm{CS}^{+} / \mathrm{US}_{\mathrm{PT}}$ and

$446 \mathrm{CS}-/ \mathrm{US}_{\mathrm{PT}}$ trials was roughly the same in both studies, while CS-only trials were unaffected by the

447 conditioning procedure. Interestingly, despite the inclusion of re-calibration procedures, a higher

448 percentage of at-pain-threshold trials, including $72 \%$ of $\mathrm{CS}^{-} / \mathrm{US}_{\mathrm{PT}}$ trials, was perceived as painful

449 in this study than in the study by Madden et al. (2016a). Nevertheless, by finding comparable

450 results while using different nociceptive stimuli (electrocutaneous vs. laser), this replication clearly 
451 strengthens the notion that classical conditioning can modulate judgements about whether an event

452 is painful or not. Furthermore, both studies used a simultaneous conditioning paradigm in which

453 CS and US were presented at the same time. This approach excludes expectation or changes in

454 arousal - to which shifts in pain thresholds have been attributed in other classical conditioning

455 studies (Harvie et al. 2016a; Miguez et al. 2014) - as the mechanism of effect. Indeed, the

456 simultaneous approach seems well suited to model chronic pain as the real-world CS (e.g. tactile

457 cue) and US (nociceptive signalling) are likely to occur at the same time (Madden et al. 2016b),

458 thus enhancing the validity of our findings. Finally, as half of our participants were not contingency

459 aware, the present study also supports the claims by Harvie et al. (Harvie et al. 2016a) and Jensen

460 et al. (Jensen et al. 2015) that expectations or conscious awareness of the learned association are

461 not required for classically conditioned shifts in pain thresholds to occur.

462 Importantly, our findings contradict those by Madden et al. (2016b), who did not find an

463 effect of classical conditioning on pain thresholds. One difference between the two studies is the

464 type of US, namely electrocutaneous versus thermal stimuli. We specifically chose to use a

465 different nociceptive stimulus than both studies by Madden and colleagues (Madden et al. 2016a;

466 Madden et al. 2016b), not only in order to investigate whether the previous findings extend to other

467 stimulus modalities, but also to help interpret their contradictory findings. The electrocutaneous

468 stimulus in the present study is considered to be less discrete than laser stimuli but more discrete

469 than contact heat stimuli (Plaghki \& Mouraux 2003). Previous investigations of conditioned fear

470 of touch confirm that the spatial precision of somatosensory cues influences the likelihood of

471 associative learning (Harvie et al. 2016b) and it seems possible that a similar mechanism is relevant

472 here. The three stimulus types have different characteristics: laser stimuli isolate nociceptors

473 (Mouraux \& Iannetti 2009) and therefore lack ecological validity, whereas the other two modalities 
474 stimulate both nociceptive and non-nociceptive mechanisms; thermal stimuli are more

475 physiological than electrocutaneous stimuli, which activate mechanoreceptors as well as

476 nociceptors but via their axons as well as receptors and nerve endings (Handwerker \& Kobal 1993),

477 but chronic pain seldom develops after repeated exposure to noxious heat stimuli. This difference

478 in discreteness between different types of nociceptive stimulation may also be a possible

479 explanation for the diverging results given by Madden et al. (2016b). One path forward that might

480 overcome these limitations is to use proprioceptive stimuli (related to movement) as the

481 conditioning stimuli (Meulders et al. 2011).

482

483

484

485

486

487

488

489

490

491

492

493

494

495

496

The present study has several limitations that require attention. First of all, participants

were frequently unsure about their indication of tactor location and many reported difficulties at the end of the experiment. In these cases, some tended to base their choice of tactor location on whether the accompanying electrocutaneous stimulus was painful or not, which resulted in location-mistakes on $12.19 \%$ of all trials, on $15.85 \%$ of all $\mathrm{CS}^{+} / \mathrm{US}_{\mathrm{PT}}$ trials and even $27.11 \%$ of all $\mathrm{CS}-/ \mathrm{US}_{\mathrm{PT}}$ trials and which might indicate a reversed form of conditioning. That is, the simultaneous presentation of vibrotactile and electrocutaneous stimuli meant that each contained information about the occurrence of the other. Consequently, the electrocutaneous stimuli may have served as CS (providing information about the tactor) on some trials. A possible explanation for this error rate might be the close proximity of the tactors. We tried to improve discriminability between tactors by increasing the distance between them from $70 \mathrm{~mm}$ (as in the study by Madden et al. 2016a) to $80 \mathrm{~mm}$, which led to fewer mistakes than in the pilot study, yet the problem still occurred occasionally. A second point of criticism is that the vibrotactors made an audible sound that could have guided participants' attention to the tactor location. Nevertheless, we decided not to use white noise to mask it because of practical reasons: the calibration procedure was not compatible with 
497 continuous white noise delivered via headphones, and switching off the white noise during

498 calibration would have formed an obvious change of context that could have interfered with

499 conditioning. Moreover, white noise would have caused additional exhaustion of the participants.

500 However, as mentioned previously, participants did not seem to be able to use the emitted sounds

501 to locate the vibrotactors, as mistakes were made nonetheless. Another limitation is that not only

502 most of the $\mathrm{CS}^{+} / \mathrm{US}_{\mathrm{PT}}$ trials but also the majority of $\mathrm{CS}^{-} / \mathrm{US}_{\mathrm{PT}}$ trials were perceived as painful. As

503 we consider the pain threshold in itself to be arbitrary (i.e., either just painful or just not painful),

504 we would expect not more than $50 \%$ of $\mathrm{CS}^{-} / \mathrm{US}_{\mathrm{PT}}$ trials to be rated as painful. The elevated 505 percentage of painful $\mathrm{CS}^{-} / \mathrm{US}_{\mathrm{PT}}$ trials may indicate a suboptimal calibration of the electrocutaneous

506 stimulus or sensitization throughout the experiment. Yet, this should not have affected the overall

507 results of our study.

508 The present findings bear important clinical implications: it is well established that classical

509 conditioning can drive pain expectancies and pain-related fear, and while such an effect has long

510 been suggested for pain itself the scientific basis for this claim has been lacking. By showing that

511 it is possible to modulate pain threshold ratings through classical conditioning in the laboratory

512 independent of the other pain-related constructs mentioned previously, it becomes more plausible

513 that similar mechanisms occur in chronic pain. Specifically, in the acute pain phase patients may

514 learn to associate the nociceptive input with other concurrent somatosensory stimuli implicitly or

515 explicitly, such that borderline painful stimulation becomes more likely to be perceived as painful

516 thus promoting maladaptive behaviours, cognitions and, eventually, chronicity.

517 Future studies to replicate and extend our findings about classical conditioning of pain

518 would be useful, as studies on this matter are still rare. Our sample was highly homogenous as it

519 consisted of healthy students, primarily females around 20 years of age. In order to draw any firm 
520 conclusions about classical conditioning of chronic pain, replication in other populations that

521 resemble demographics of individuals suffering from chronic pain more closely is necessary.

522 Additionally, the present study focused on discrete locations of stimulation, but it might be

523 valuable to investigate whether the conditioned shift in report of pain thresholds does also

524 generalize to proximal or distal areas, in order to better understand the spreading of pain.

525 Furthermore, as mentioned previously, it would be interesting to investigate whether the

526 discreteness of the US affects pain conditioning by directly comparing the different types of

527 nociceptive stimulation. Lastly, future studies may include psychophysiological measures in

528 addition to self-reports of pain to get a more complete picture of the effects of pairing noxious and

529 non-noxious stimuli on pain perception and other pain-related outcomes.

530

531

\section{Conclusion}

532

This study provides evidence that ratings of at-pain-threshold electrocutaneous stimuli can 533 be modified through classical conditioning. Our results, therefore, advance the debate about 534 whether or not pain itself can be classically conditioned and, although the study was conducted in 535 healthy participants, it contributes to our understanding of the development and maintenance of 536 chronic pain. From a scientific perspective, classical conditioning may pose a useful method for 537 modelling chronic pain in healthy participants. Furthermore, against the backdrop of broad 538 scientific knowledge about classical conditioning in other psychopathologies (Breivik 2016), the 539 present findings suggest that this line of research bears great potential to improve interventions 540 and preventive measures against chronic pain in the future. 
543 The results of this study have been presented at the Pain Research Meeting, Antwerp (Belgium),

544 18-19 September 2017, for which JT received the "best presentation" prize. The authors would 545 like to thank Jeroen Clarysse for his technical support. 


\section{References}

548 Abraira VE, and Ginty DD. 2013. The sensory neurons of touch. Neuron 79:618-639.

549 Bache SM, and Wickham H. 2014. magrittr: a forward-pipe operator for R. $R$ package version 1.

550 Brandt MJ, IJzerman H, Dijksterhuis A, Farach FJ, Geller J, Giner-Sorolla R, Grange JA, Perugini

551 M, Spies JR, and Van't Veer A. 2014. The replication recipe: What makes for a convincing 552 replication? Journal of Experimental Social Psychology 50:217-224.

553 Bräscher A-K, Kleinböhl D, Hölzl R, and Becker S. 2017. Differential classical conditioning of the nocebo effect: increasing heat-pain perception without verbal suggestions. Frontiers in

Breivik H. 2016. Associative learning mechanisms may trigger increased burden of chronic pain; unlearning and extinguishing learned maladaptive responses should help chronic pain patients. Scandinavian Journal of Pain 11:34-35. 10.1016/j.sjpain.2015.11.012

Claes N, Vlaeyen JW, and Crombez G. 2016. Pain in context: Cues predicting a reward decrease fear of movement related pain and avoidance behavior. Behaviour Research and Therapy $84: 35-44$.

Crawford JR, and Henry JD. 2004. The Positive and Negative Affect Schedule (PANAS): Construct validity, measurement properties and normative data in a large non-clinical sample. British Journal of Clinical Psychology 43:245-265.

Domjan M. 2005. Pavlovian conditioning: a functional perspective. Annual Review of Psychology $56: 179-206$.

567 Faul F, Erdfelder E, Lang A-G, and Buchner A. 2007. G* Power 3: A flexible statistical power analysis program for the social, behavioral, and biomedical sciences. Behavior Research Methods 39:175-191. 
570 Flor H, and Birbaumer N. 1994. Acquisition of chronic pain: Psychophysiological mechanisms.

571

572 Gramsch C, Kattoor J, Icenhour A, Forsting M, Schedlowski M, Gizewski ER, and Elsenbruch S. 2014. Learning pain-related fear: neural mechanisms mediating rapid differential conditioning, extinction and reinstatement processes in human visceral pain. Neurobiology of Learning and Memory 116:36-45.

Handwerker H, and Kobal G. 1993. Psychophysiology of experimentally induced pain. Physiological reviews 73:639-671.

Harvie DS, Meulders A, Madden VJ, Hillier SL, Peto DK, Brinkworth R, and Moseley GL. 2016a. When touch predicts pain: predictive tactile cues modulate perceived intensity of painful stimulation independent of expectancy. Scandinavian Journal of Pain 11:11-18.

Harvie DS, Meulders A, Reid E, Camfferman D, Brinkworth RS, and Moseley GL. 2016 b. Selectivity of conditioned fear of touch is modulated by somatosensory precision. Psychophysiology 53:921-929.

Inui K, Tran TD, Hoshiyama M, and Kakigi R. 2002. Preferential stimulation of A $\delta$ fibers by intraepidermal needle electrode in humans. Pain 96:247-252.

Jensen K, Kirsch I, Odmalm S, Kaptchuk TJ, and Ingvar M. 2015. Classical conditioning of analgesic and hyperalgesic pain responses without conscious awareness. Proceedings of the National Academy of Sciences 112:7863-7867.

Jepma M, and Wager TD. 2015. Conceptual conditioning: mechanisms mediating conditioning effects on pain. Psychological Science 26:1728-1739. 
591 Klinger R, Matter N, Kothe R, Dahme B, Hofmann UG, and Krug F. 2010. Unconditioned and 592 conditioned muscular responses in patients with chronic back pain and chronic tension593 type headaches and in healthy controls. Pain 150:66-74.

594

595

596

597

598

599

600

601

602

603

604

605

606

607

608

609

610

611 Progress in Behavior Modification 18:1-42.

Madden VJ, Bellan V, Russek LN, Camfferman D, Vlaeyen JW, and Moseley GL. 2016a. Pain by association? Experimental modulation of human pain thresholds using classical conditioning. Journal of Pain 17:1105-1115.

Madden VJ, Harvie DS, Parker R, Jensen KB, Vlaeyen JW, Moseley GL, and Stanton TR. 2015. Can pain or hyperalgesia be a classically conditioned response in humans? A systematic review and meta-analysis. Pain Medicine:pnv044.

Madden VJ, Kamerman P, Bellan V, Catley MJ, Russek LN, Camfferman D, and Moseley GL. April, 2018. "The stimulus-response characteristics of the Sensation and Pain Rating Scale (SPARS) for rating non-painful and painful experiences". Poster, 2018 Australian Pain Society 38th and New Zealand Pain Society Conjoint Annual Scientific Meeting. Sydney, Australia.

Madden VJ, and Moseley GL. 2016. Do clinicians think that pain can be a classically conditioned response to a non-noxious stimulus? Manual Therapy 22:165-173.

Madden VJ, Russek LN, Harvie DS, Vlaeyen JW, and Moseley GL. 2016b. Classical Conditioning Fails to Elicit Allodynia in an Experimental Study with Healthy Humans. Pain Medicine:pnw221. 
612 Meulders A, Vansteenwegen D, and Vlaeyen JW. 2011. The acquisition of fear of movement613 related pain and associative learning: a novel pain-relevant human fear conditioning

614 paradigm. Pain 152:2460-2469.

615 Miguez G, Laborda MA, and Miller RR. 2014. Classical conditioning and pain: conditioned 616 analgesia and hyperalgesia. Acta Psychologica 145:10-20.

617

618

619

620

621

622

623

624

625

626

627

628

629

630

631

632 pain. Pain 156:35-38.

Mouraux A, De Paepe AL, Marot E, Plaghki L, Iannetti GD, and Legrain V. 2013. Unmasking the obligatory components of nociceptive event-related brain potentials. Journal of Neurophysiology 110:2312-2324. 10.1152/jn.00137.2013

Mouraux A, and Iannetti GD. 2009. Nociceptive laser-evoked brain potentials do not reflect nociceptive-specific neural activity. Journal of Neurophysiology 101:3258-3269.

Mouraux A, Marot E, and Legrain V. 2014. Short trains of intra-epidermal electrical stimulation to elicit reliable behavioral and electrophysiological responses to the selective activation of nociceptors in humans. Neuroscience Letters 561:69-73.

Peuterl S, Diestl I, Vansteenwegenl D, Berghl O, and Vlaeyenl JW. 2011. Understanding fear of pain in chronic pain: interoceptive fear conditioning as a novel approach. European Journal of Pain 15:889-894.

Plaghki L, and Mouraux A. 2003. How do we selectively activate skin nociceptors with a high power infrared laser? Physiology and biophysics of laser stimulation. Clinical Neurophysiology 33:269-277. 
633 Watson D, Clark LA, and Tellegen A. 1988. Development and validation of brief measures of 634 positive and negative affect: the PANAS scales. Journal of Personality and Social $635 \quad$ Psychology 54:1063.

636 Wickham H. 2009. ggplot2: Elegant Graphics for Data Analysis Springer-Verlag New York. 637 Version.

638 Wickham H. 2017. Tidyverse: Easily install and load'tidyverse'packages. $R$ package version 1.

639 Wickham H, Hester J, Francois R, Jylänki J, and Jørgensen M. 2017. readr: read rectangular text 640 data. R package version 1.1. 1.

641 Williams AE, and Rhudy JL. 2007. The influence of conditioned fear on human pain thresholds: 642 does preparedness play a role? Journal of Pain 8:598-606.

643 Zaman J, Vlaeyen JW, Van Oudenhove L, Wiech K, and Van Diest I. 2015. Associative fear 644 learning and perceptual discrimination: a perceptual pathway in the development of chronic 645 pain. Neuroscience \& Biobehavioral Reviews 51:118-125.

646 
Figure 1

Set-up of vibrotactors and the electrodes on the back (adapted with permission from Madden et al. 2016a).
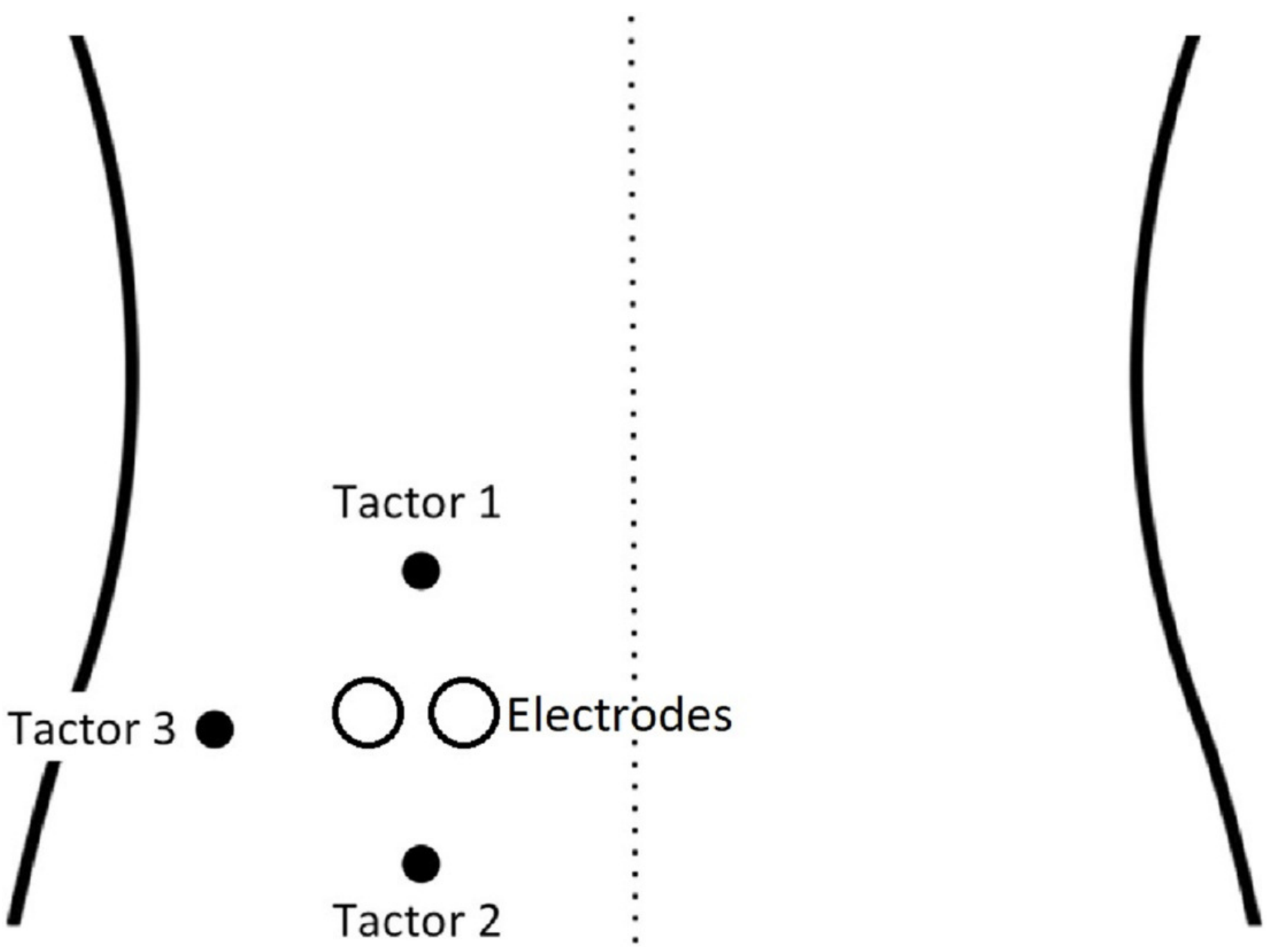
Figure 2

The four phases of the experimental procedure.

Dots indicate vibrotactile stimuli, dark dots show which vibrotactor was activated for each trial type. Circles indicate the field on which the two electrodes were placed (adapted with permission from Madden et al. 2016a).

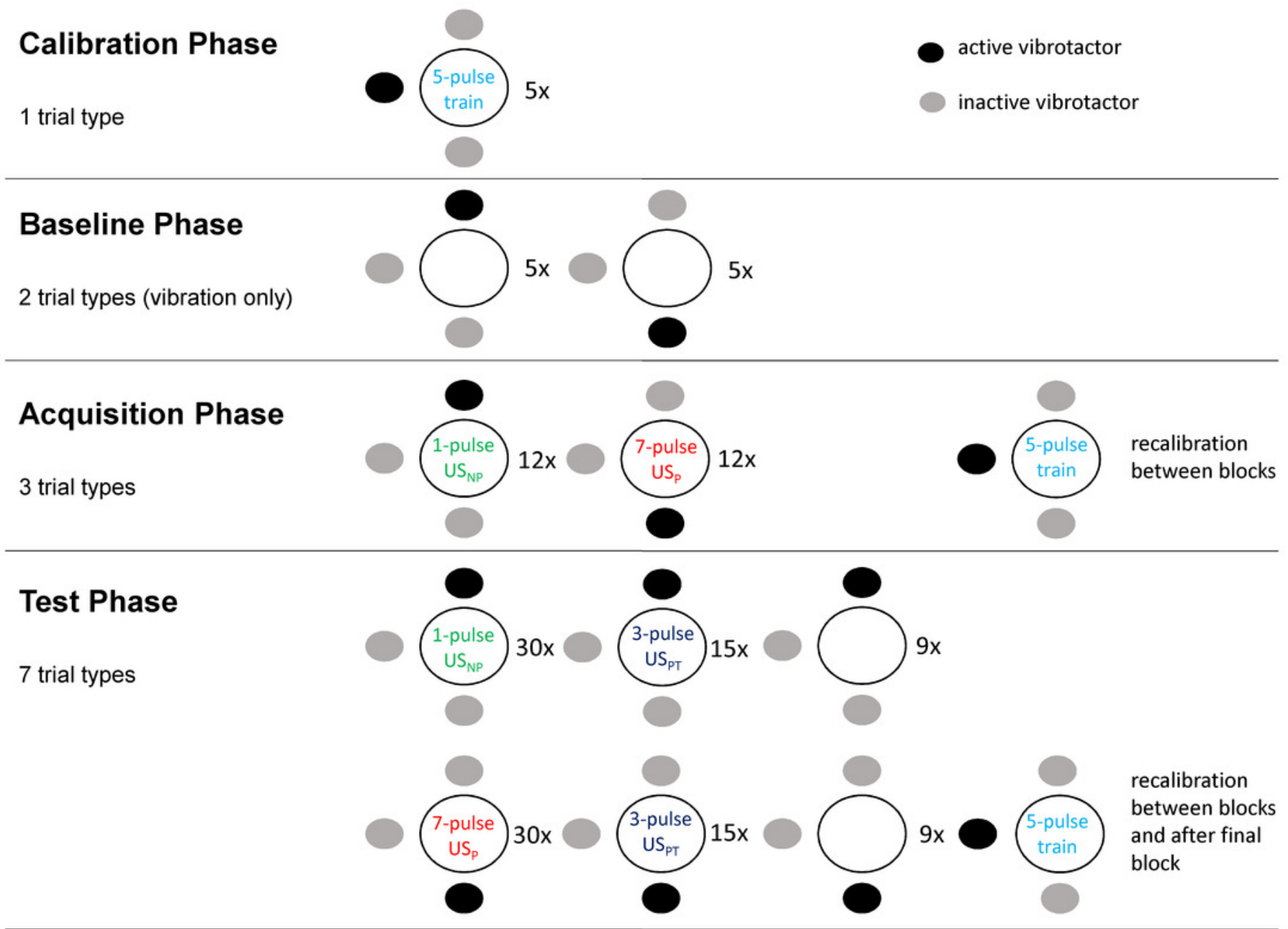


Figure 3

Scatter boxplot of expectancy ratings by CS type across baseline, acquisition and test phases (mean, standard error), panelled by contingency awareness.

Expectancy ratings by CS type, paneled by contingency awareness

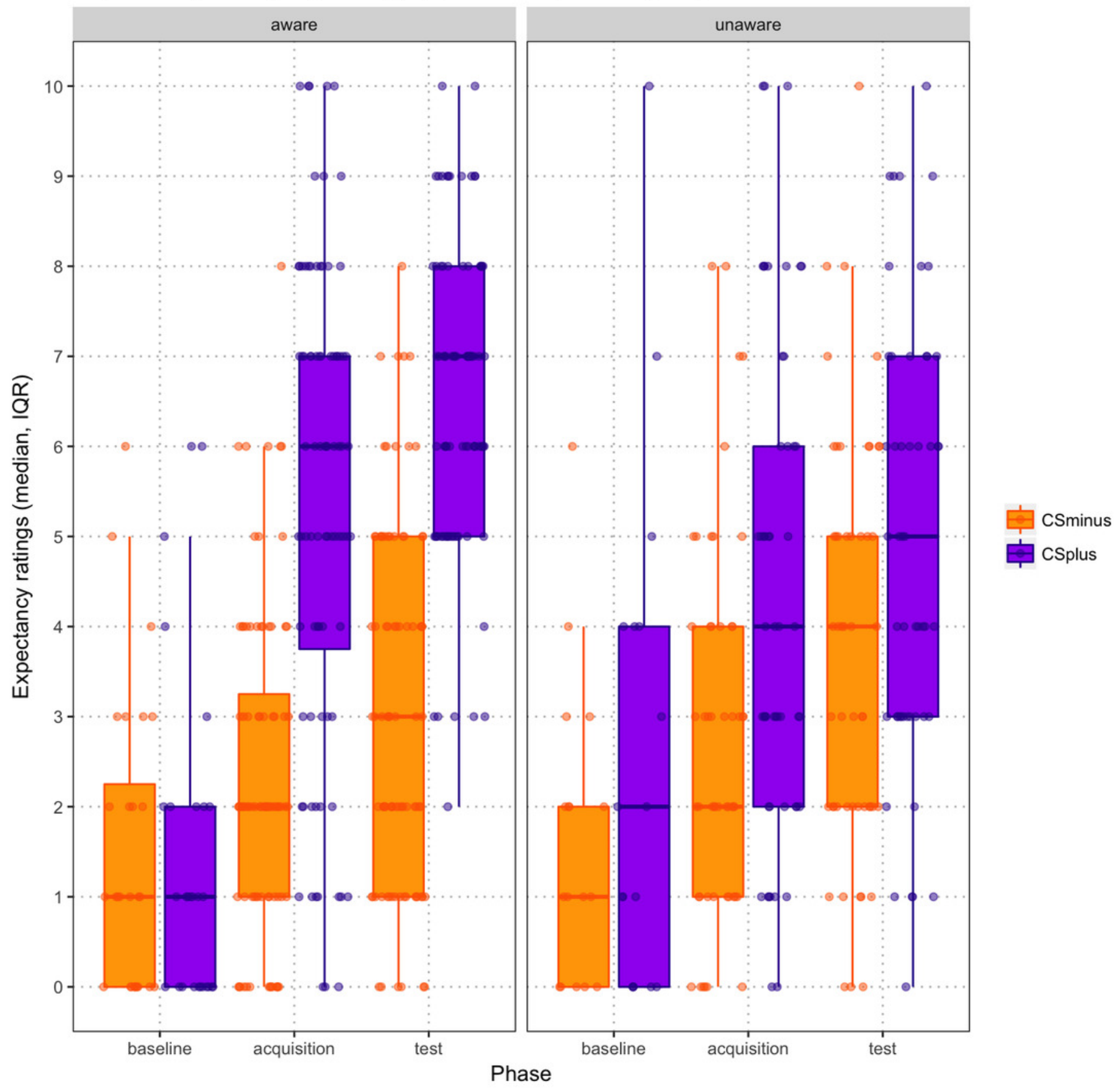


Figure 4

Scatter boxplots of exact mean intensity ratings (error bars = standard error) by CS type during acquisition and test phase.

Trials intensity rating by CS type

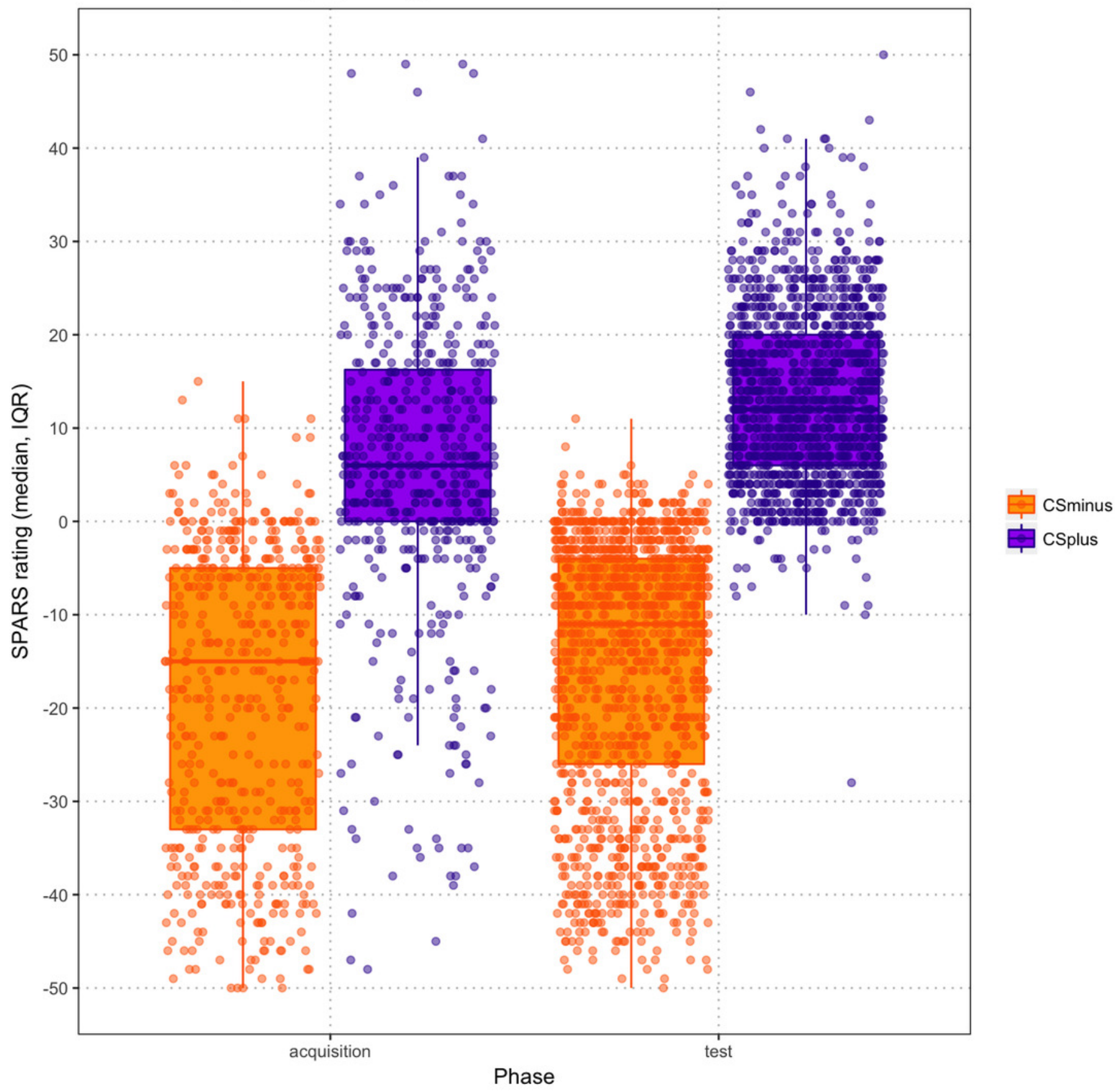




\section{Table $\mathbf{1}$ (on next page)}

Participant characteristics $(n=45)$.

${ }^{1}$ Clinical meaning of PANAS scores based on Crawford and Henry (2004). 
1

\begin{tabular}{lcc}
\hline Outcome & Mean (SD) & Range \\
\hline Age & $19.44(2.668)$ & $18-31$ \\
Positive state affect (PANAS) & $26.69(6.657)$ & $14-39$ (normal range) $^{1}$ \\
Negative state affect (PANAS) & $13.36(2.647)$ & $10-19$ (normal range) $^{1}$
\end{tabular}

2 


\section{Table 2 (on next page)}

Mean stimulus intensity ratings (SD) for the three phases.

$\mathrm{CS}=$ conditioned stimulus; $\mathrm{US}_{\mathrm{P}}=$ painful unconditioned stimulus; $\mathrm{US}_{\mathrm{NP}}=$ non-painful unconditioned stimulus; $U_{\mathrm{PT}}=$ at-threshold unconditioned stimulus. 
1

\begin{tabular}{|c|c|c|c|}
\hline & Baseline phase & Acquisition phase & Test phase \\
\hline $\mathrm{CS}+/ \mathrm{US}_{\mathrm{P}}$ & & $3.722(1.862)$ & $12.213(1.562)$ \\
\hline $\mathrm{CS}-/ \mathrm{US}_{\mathrm{NP}}$ & & $-19.181(2.859)$ & $-13.528(2.376)$ \\
\hline $\mathrm{CS}+/ \mathrm{US}_{\mathrm{PT}}$ & & & $6.174(5.109)$ \\
\hline CS-/US & & & $3.596(3.714)$ \\
\hline $\mathrm{CS}+$ only & $-32.811(3.150)$ & & $-24.821(15.464)$ \\
\hline CS- only & $-35.489(2.822)$ & & $-27.222(12.992)$ \\
\hline
\end{tabular}

2 